\title{
Enhanced Recovery After Surgery
}

\author{
Nicole Greenshields ${ }^{1,2} \cdot$ Monty Mythen ${ }^{3}$
}

Published online: 27 January 2020

(C) The Author(s) 2020

\begin{abstract}
Purpose of Review To provide a brief summary of the progress of enhanced recovery over the last 5 to 10 years and set out what the future might hold for such programmes.

Recent Findings There has been significant progress in the adoption of enhanced recovery programmes across multiple surgical specialities. However there is still difficulty in sustaining a target of $>80 \%$ compliance, which has been identified through research as the optimal level to observe best patient results. With increasing interest in perioperative medicine and prehabilitation, more focus is being put into enhanced recovery programmes.

Summary The evidence continues to support the use of enhanced recovery programmes to reduce patient mortality, morbidity and length of stay and therefore saving cost and resource. However more progress needs to be made in adoption and compliance to these programmes. In the future, advances in technology may aid programme implementation and data collection.
\end{abstract}

Keywords Enhanced $\cdot$ Recovery $\cdot$ ERAS $\cdot$ Perioperative $\cdot$ Prehabilitation

\section{Introduction}

Kehlet, a colorectal surgeon, published a paper in the British Journal of Anaesthesia in 1997 first introducing the concept of enhanced recovery. Kehlet described a "multimodal approach to control post-operative pathophysiology and rehabilitation" [1]. This seminal paper suggested the use of evidence-based interventions targeted to the preoperative, intraoperative and post-operative periods to improve patient outcomes. Most of these techniques still form the key elements of enhanced recovery pathways today. The key aims were to reduce postoperative organ dysfunction through minimizing the surgical stress response and therefore reducing morbidity, accelerating

This article is part of the Topical Collection on Preoperative Evaluation

Nicole Greenshields

greenshieldsn@gmail.com

Monty Mythen

M.mythen@ucl.ac.uk

1 University College London, Gower St, Bloomsbury, London WC1E 6BT, UK

2 Division of Surgery and Interventional Science, University College London, London, England, UK

3 Anaesthesia and Critical Care, University College London, Gower St, Bloomsbury, London WC1E 6BT, UK recovery, reducing hospital stays and reducing overall costs. In 2001, Kehlet published a subsequent paper with Wilmore [2] describing "fast-track surgery", now known as enhanced recovery. They described evidence-based interventions in four strands as outlined below:

\section{Preoperative}

- Optimisation of medical conditions

- Cessation of alcohol and smoking

- Patient education

\section{Stress Reduction}

- Regional anaesthesia and opioid sparing techniques

- Minimally invasive operative techniques

- Normothermia

- Pharmacological modifiers

\section{Post-Operative}

- Minimization of nasogastric tubes and drains

- Early mobilization

- Early oral nutrition 
- Pain relief

- Prevention and treatment of nausea and vomiting

In the same year, the enhanced recovery after surgery (ERAS) society and study group was founded [3], and its research suggested that there was a large variation in actual practice compared to what was deemed best practice in the literature. The first evidence-based multimodal ERAS protocol for colorectal surgery was subsequently published in 2005 [4]. In 2007 it was noted that having one of these protocols is not enough to ensure compliance and adherence [5] and that better organization of care and more experience is required (a problem that is ongoing today). The ERAS Society has published a range of guidelines for ERAS pathways for different surgical specialities including cardiac, thoracic, colorectal, breast, gynaecology, upper gastrointestinal and urology [6••].

The Enhanced Recovery Partnership Programme was launched in 2009 to work as clinical champions to engage medical teams and support implementation of ERAS programmes in the English National Health Service (NHS). During this period, national data showed a reduction in length of stay (LOS) for specialities where ERAS programmes were used. This concluded with the Department of Health report in 2011 [7], which looked at the implementation of enhanced recovery programmes in colorectal, musculoskeletal, gynaecology and urology surgeries. While there was significant progress in increasing the use of the programmes over the 3 -year study, with $86 \%$ of centres having at least one programme, the report concluded that ongoing support was needed to maintain momentum.

Over the same period of 2009-2012, the data from the Enhanced Recovery Partnership Programme was published [8]. This demonstrated that a high programme compliance was required in order to see benefits. In particular, a doseresponse effect was identified, where shorter LOS was seen with higher compliance to the pathway. A compliance level of $80 \%$ was needed in colorectal and urological pathways to see statistically significant results, which supported the idea that consistent protocolised pathways provide better patient outcomes. The literature supports the relationship with ERAS and a reduction in complications, mortality and length of stay and therefore more cost savings $[9,10]$.

\section{Protocolised Pathways}

There are several areas of medicine where discrepancies in medical care have been noted and protocolised pathways subsequently introduced to improve and standardize the care.

\section{National Emergency Laparotomy Audit}

The United Kingdom's (UK) National Emergency Laparotomy Audit (NELA) is led by the Health Services Research Centre
(HSRC) and Royal College of Anaesthetists (RCoA), with input from the Royal College of Surgeons (RCS) and the Healthcare Quality Improvement Partnership (HQIP). It examines patients undergoing emergency surgery and was launched in 2011 following evidence that there was a large variation in mortality and provision of care for patient having emergency laparotomies. To date there have been four reports produced by NELA, the most recent [11] highlighting a reduction in hospital stay as well as mortality. Over the past 4 years, the 30-day mortality has reduced from $11.8 \%$ to $9.4 \%$, with 700 fewer patient deaths a year. There has also been a significant reduction in hospital stay from 19.2 days to 15.6 days saving the NHS more than $£ 34 \mathrm{~m}^{11}$.

Areas identified for improvement include time for septic patients to receive antibiotics, geriatrician involvement for patients over the age of 70 and consultant presence outside of usual hours. The concepts of the enhanced recovery programme can be applied to emergency surgery. Early mobilization, minimally invasive surgery, reduction in drains and nasogastric tubes benefit the emergency surgical patient. The key papers that form the standards of care for the NELA patient are available on its website, beginning with the RCS report highlighting the importance of quantifying the risk for each individual surgical patient and outlining standards to improve care [12-14]. As healthcare progresses and the age of operative patients are higher [15], we as a profession need to consider the provision of emergency and elective healthcare. Functionality has been added to quarterly update reports and allows one's own unit comparisons to national figures, an important tool for benchmarking how a centre is doing.

\section{Fractured Neck of Femur}

The UK's National Hip Fracture Database (NHFD) [16] provides information for those having orthopaedic surgery for fractured neck of femur and was established in 2007. NHFD provides online hospital dashboards, progress reports and ratings. This allows comparison between NHS Trusts and uses a visual traffic light rating system for adherence to guidelines. The most recent report showed that $78.8 \%$ of patients were out of bed the day after surgery which supported early mobilization [17]. NHFD's most recent focus has been the importance of ortho-geriatric assessment, a type of preoperative assessment, similar to the ERAS principles.

\section{Perioperative Quality Improvement Programme}

The UK's Perioperative Quality Improvement Programme (PQIP) was set up in 2016 [18] by the National Institute of Academy of Anaesthesia (NIAA) and RCoA in collaboration with RCS, Royal College of Physicians (RCP), Royal College of Nursing $(\mathrm{RCoN})$, Faculty of Intensive Care Medicine (FICM) and the Faculty of Pain Medicine (FPM). This programme is focused on major noncardiac surgery outcomes 
such as complication rates, "failure to rescue" and the important patient-reported outcome measures (PROMs). The aim is to improve outcomes by reducing variations in care and supporting the implementation of best practice. The first annual report was published in April 2018 [19], and PQIP is rapidly expanding to include Wales and Scotland as well as orthopaedic and plastic surgeries.

Five key improvement opportunities were identified for the upcoming year (2018-2019) with themes common to ERAS programmes as follows:

- Preoperative management of diabetes and anaemia

- Individualized risk assessment

- Enhanced recovery and sharing of pathways among hospitals

- Individualized pain management, using multimodal strategies including regional anaesthesia

- Drinking, eating and mobilizing (DrEaMing); empowering patients through preparation and use of patient diaries

In the ERAS section of the PQIP report [19] and visualized in Fig. 1 is breakdown of patients and whether they are on an enhanced recovery pathway or not. Between 40 and $73 \%$ of the elective patients were on an enhanced recovery programme, with a national average of $61.4 \%$. However there was large variation among hospitals, with one hospital having all patients enrolled and one having not a single patient enrolled.

Again this research highlighted large variations in care across different sites. Only two out of the ten ERAS components had a greater than $80 \%$ compliance within colorectal surgery (see Table 1).

The second report [20 $0^{\bullet}$ includes enhanced recovery as one if its top priorities for 2019-2020. In particular, the elements that PQIP wants to focus on are as follows:

- Surgery schools or other tailored preparations, where patients attend a group session with members of the multidisciplinary team preoperatively to be educated on the anaesthetic, surgery and recovery
Table 1 National compliance with enhanced recovery principals from the UK's PQIP report [19].

\begin{tabular}{ll}
\hline ERAS component & $\%$ compliance \\
\hline Carbohydrate preloading & 47 \\
Individualized risk assessment & 67 \\
Warming devise used & 97 \\
Cardiac output monitor used & 31 \\
No nasogastric tube in recovery (colorectal only) & 91 \\
No drains in recovery (colorectal only) & 57 \\
Drinking at 24 h & 78 \\
Eating at 24 h & 60 \\
Mobilizing at 24 h & 76 \\
DrEaMing (on day 1) & 53 \\
\hline
\end{tabular}

- Preoperative nutritional assessment, carbohydrate loading and minimizing starvation

- Drinking, eating (for nutritional supplementation) and mobilizing within $24 \mathrm{~h}$

- Minimize tubes, drains and "institutionalization"

A new pilot project within PQIP is called the perioperative medicine Variable Life Adjusted Display (pomVLAD) [21], and is intended to provide a real time risk adjusted postoperative morbidity measure via online dashboards and regular reports. The dashboard will show the observed and expected post-operative morbidity and will be combined with the ERAS recommendation reporting. It will also show areas where local quality improvement is needed.

\section{Perioperative Medicine}

In January 2015, the RCoA launched its vision document: "Perioperative Medicine: the pathway to better surgical care [22••]", with the aim to develop a collaborative programme for perioperative care delivery. Within the statement of support, the phrase "encompasses the principles of enhanced recovery that we know can deliver better patient experience as well as a more efficient elective care pathway and can lead to the
Fig. 1 PQIP patients and enhanced recovery [19]

Patients in green: on enhanced recovery (ER) pathway. Patients in red: not on ER pathway. Blue: unknown. Reproduced here with permission from the Royal College of Anaesthetists, but the $\mathrm{RCoA}$ has not reviewed this as a whole. Originally published in the PQIP Annual Report 2017-18.

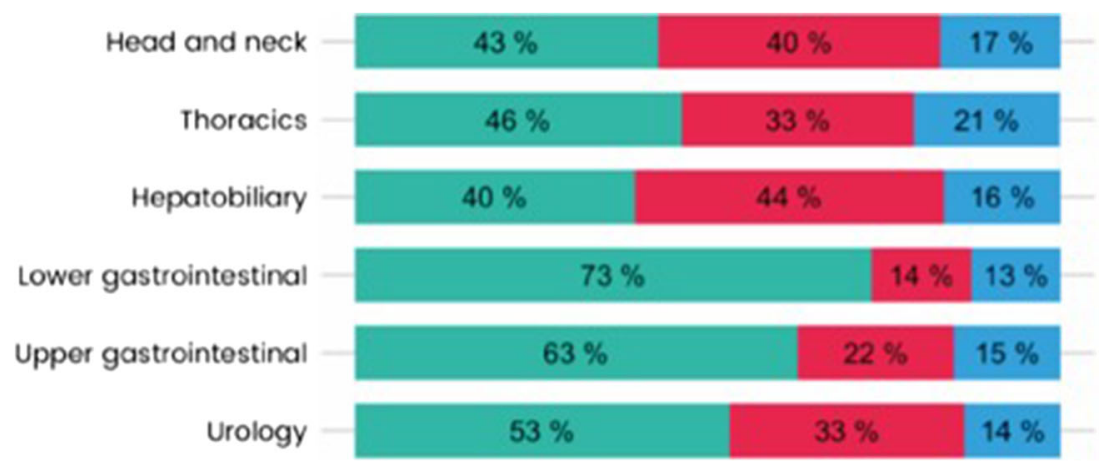


delivery of the best possible quality of care for patients". Enhanced recovery programmes are at the centre of this agenda. The RCOA has subsequently produced an educational film for both patients and health professionals in order to explain the concept of the suggested care model [23]. Alongside the launch of this, we have seen the addition of perioperative learning outcomes as a compulsory module for trainee anaesthetists to support the future delivery of perioperative care.

\section{Cardiac ERAS}

The ERAS Society and the United States (US)-based ERAS Cardiac Society jointly produced evidence-based guidelines to standardize care and improve outcomes for cardiac surgery [ 24]. This particular ERAS programme advocates for prehabilitation in the form of education, nutritional, exercise, social support and anxiety reduction. While this is a recommendation, the authors note that the supporting evidence is limited.

\section{Centre for Perioperative Care}

The UK's Centre for Perioperative Care (CPOC) was launched in May 2019 as a partnership of the RCoA, RCS, RCP, RCoN, Royal College of General Practitioners and the Association of Anaesthetists. The collaboration of these varied organizations strongly demonstrates the importance of the multidisciplinary team. The aim of the centre is to drive integration of multidisciplinary cross-organizational patient care across the UK through the "triple aim" [25] of:

- Improving quality of healthcare

- Improving health of the population

- Achieving value and financial stability

Included in one of its initial focus points is enhanced recovery, along with; management of diabetes and anaemia, individualized pain management and DrEaMing. These aims are streamlined with the PQIP aims for the upcoming year. This demonstrates that enhanced recovery is still very much at the forefront of delivering good quality perioperative care. The CPOC believes that working with patients and the public at all stages of care is vital to provide the best quality for patients. In time, PQIP will be able to provide a wealth of data to understand the progress of major surgery and the advances CPOC is making.

\section{Outcome Measures}

While the initial literature on ERAS programmes focused on standard measures such as mortality, morbidity and readmission rates, there is an increasing need for the profession to be focussing more on PROMs [26]. The important measures for patients are generally their quality of life or degree of independence. While standard measures are still important, over the last 10 years, there has been a gradual movement towards using PROMs and ensuring other outcome measures are transparent.

The NHS has been part of this change to look at PROMs. Since 2009 the NHS has been collecting data on hip and knee replacements, in particular PROMs, which are available via NHS digital [26]. PROMs can be categorized as [27]:

- Patient satisfaction

- Decision regret

- Patient preference

- Health-related quality of life

PQIP, for example, collects data on generic health pre- and post-operatively via EuroQol's health-related quality of life score (EQ-5D) [28] and disability assessment pre- and postoperatively via the World Health Organization's Disability Assessment Score (WHOSDAS) [29]. This identifies important data on how patients are doing functionally, compared to their baseline. This can help with difficult decisions such as if to operate.

\section{Beyond ERAS}

\section{ERAS Plus}

ERAS plus [30] has been developed and is currently piloting in the UK's Manchester area. The key patient's aims are getting active, chest training, muscle strengthening, surgery school, living, and eating well. Provisional results show a $50 \%$ reduction in post-operative pulmonary complications, an average LOS reduction by 3 days, and trust financial savings of $£ 500,000$ per year [31]. The group has developed a mobile phone application; the ERAS plus app - giving exercise examples, links to local gyms, information on eating well and advice on giving up smoking and alcohol. With more people engaging in technology - irrespective of age or generation - such applications will undoubtedly form vital resources going forward.

\section{Prehabilitation}

The concept of prehabilitation originated from the oncological speciality, and given its close relationship with surgery has rapidly progressed. In July 2019 , the Principals and Guidance for Prehabilitation Within the Management and Support of People with Cancer document was launched by the UK's Macmillan Cancer Care and the National Institute 
of Health Research [32•]. It focused on preparing for treatment by promoting healthy behaviours as well as exercise, nutrition and physiological interventions. It has been previously believed that the urgency of surgery in oncology meant there was not enough time for preoperative interventions. However it has been shown as little as 2 weeks of interventions can improve outcomes [33•]. There is emerging evidence to support that all of the individual aspects of prehabilitation provide better patient outcomes [34•]:

- Psychosocial interventions reduce psychological distress as well as function impairment $[35,36]$

- Physical exercise reduces post-operative complications and length of stay [37-39].

- Nutrition improves response to treatment [40]

It is therefore not unreasonable to consider prehabilitation as an extension of enhanced recovery, and in a good enhanced recovery programme, there is a focus on preoperative nutrition, exercise and optimisation of medical conditions. It is also important to note that there are benefits from prehabilitation even if the surgical option is not the preferred option after patient-centred discussions. For example, evidence shows patients will return to a better functional status and quality of life following chemotherapy if they have had prehabilitation compared to those who have not [33•].

\section{Technology and Healthcare}

There is a current trend of the public wearing and using technological devices that track fitness (e.g. using a smart phone to check how many steps a patient is doing in a day), with an inbuilt target of 10,000 steps a day. Separately the US Food and Drug Administrations (FDA) approved an iPhone application that records the equivalent of lead 1 of an electrocardiogram, for informational use only. Some private healthcare providers (such as vitality) advocate the use of step counters in order for customers to reduce their insurance premiums, with the assumption if you are more active, you are less likely to have health problems and also collect data on food shopping habits via supermarket loyalty cards. Therefore it is unsurprising that technology is starting to feature more in literature and research findings.

One study [41] provided its patients with a Fitbit Charge $\mathrm{HR}^{\mathrm{TM}}$, (Fitbit, San Francisco, CA) and found that these devices are good at capturing biophysical parameters and associated with high-patient engagement in healthcare. While the devices were able to assess sleep as well as general activity, the integration and merging of data into existing care pathways and databases is more challenging. More recently, one group found patients with a higher post-operative daily step count were less likely to have a prolonged LOS, allowing prediction of those at risk of poor outcomes [42].
Portable laptops and tablets are becoming more popular within NHS hospitals. There is increasing transition to paperless systems with electronic health records, and the advantages of this for real-time data collection are huge.

\section{Conclusions}

In order to provide optimal patient care, patients should be placed on an enhanced recovery programme post-surgery. While there are concerns over a "one size fits all" approach, a multitude of evidence-based interventions are consistently shown to benefit patients. Given the potential since the launch of the UK's enhanced recovery partnership in 2009 [8], one would expect for most hospitals to be now using ERAS as a standard of care for most surgeries. However as PQIP [19] has demonstrated in their most recent report, this is not the case.

The biggest challenges within ERAS are data collection and looking at compliance with pathways and patient outcomes. As the NHS enhances its digital capabilities, the ease of data collection should improve. Alongside this, larger databases such as PQIP will continue to provide a rich data set for analysis, and almost all surgical specialties now have a published enhanced recovery protocol. With the ability to compare outcomes, it creates the opportunity to share protocols among hospitals so that all the programmes that work well can be disseminated.

A credible goal for the profession will be to implement real-time data collection and analysis. Technology will play a significant part in supporting this, alongside an increasing level of patient engagement with wearable devices. From the medical perspective, having real-time compliance rates to the components of the pathway provide a visual representation of whether or not aims are met. Where aims are not, this can be acted on in a timely fashion, and such feedback provides early reinforcement to entrench positive behaviours. One of the reasons for launch of NELA and enhanced recovery programmes was due to the large variations in outcomes among centres; these online databases allow direct comparisons and highlight areas for improvement.

With increasing awareness and knowledge of perioperative medicine and the establishment of the CPOC, as well as the emerging evidence on prehabilitation, there is new invigorated energy to ensure more enhanced recovery protocols are instigated and complied to. The authors hope that in a further 5 years, all elective surgical patients will be on enhanced recovery programmes.

\section{Compliance with Ethical Standards}

Conflict of Interest Nicole Greenshields declares that she has no conflict of interest. 
Monty Mythen receives research funding from Smiths Medical as an Endowed University Chair; has received compensation from Edwards Lifesciences And Baxter for service as a consultant; is the Founding Editor-In-Chief Of TopMedTalk, Founding Editor Of Perioperative Medicine, and is a member of the Editorial Board for The British Journal Of Anaesthesia.

Human and Animal Rights and Informed Consent All reported studies/ experiments with human or animal subjects performed by the authors have been previously published and complied with all applicable ethical standards (including the Helsinki declaration and its amendments, institutional/national research, committee standards, and international/national/institutional guidelines.

Open Access This article is licensed under a Creative Commons Attribution 4.0 International License, which permits use, sharing, adaptation, distribution and reproduction in any medium or format, as long as you give appropriate credit to the original author(s) and the source, provide a link to the Creative Commons licence, and indicate if changes were made. The images or other third party material in this article are included in the article's Creative Commons licence, unless indicated otherwise in a credit line to the material. If material is not included in the article's Creative Commons licence and your intended use is not permitted by statutory regulation or exceeds the permitted use, you will need to obtain permission directly from the copyright holder. To view a copy of this licence, visit http://creativecommons.org/licenses/by/4.0/.

\section{References}

Papers of particular interest, published recently, have been highlighted as:

- Of importance

•- Of major importance

1. Kehlet H. Multimodal approach to control postoperative pathophysiology and rehabilitation. Br J Anaesth. 1997;78(5):606-17. https://doi.org/10.1093/bja/78.5.606.

2. Wilmore DW, Kehlet H. Management of patients in fast track surgery. BMJ. 2001;322(7284):473-6. https://doi.org/10.1136/bmj. 322.7284.473.

3. ERAS Society. http://erassociety.org/about/history/. Accessed 10th Aug 2019.

4. Fearon KCH, Ljungqvist O, Von Meyenfeldt M, Revhaug A, Dejong CHC, Lassen K, et al. Enhanced recovery after surgery: a consensus review of clinical care for patients undergoing colonic resection. Clin Nutr. 2005;24(3):466-77. https://doi.org/10.1016/j. clnu.2005.02.002.

5. Maessen J, Dejong CHC, Hausel J, Nygren J, Lassen K, Andersen $\mathrm{J}$, et al. A protocol is not enough to implement an enhanced recovery programme for colorectal resection. Br J Surg. 2007;94(2):22431. https://doi.org/10.1002/bjs.5468.

6.• ERAS Society Guidelines. http://erassociety.org/guidelines/list-ofguidelines/ Accessed 10th Aug 2019. Links to all published enhanced recovery society guidelines.

7. Enhanced Recovery Partnership Programme. Delivering enhanced recovery-helping patients to get better sooner after surgery. London: Department of Health; 2010.

8. Simpson JC, Moonesinghe SR, Grocott MPW, Kuper M, McMeeking A, Oliver CM, et al. Enhanced recovery from surgery in the UK: an audit of the enhanced recovery partnership programme 2009-2012†. Br J Anaesth. 2015;115(4):560-8. https://doi.org/10.1093/bja/aev105.

9. Li L, Jin J, Min S, Liu D, Liu L. Compliance with the enhanced recovery after surgery protocol and prognosis after colorectal cancer surgery: A prospective cohort study [published correction appears in Oncotarget. 2017 Oct 27;8(52):90605]. Oncotarget. 2017;8(32):53531-53541. Published 2017 Jun 22. https://doi.org/ 10.18632/oncotarget.18602.

10. Berian JR, Ban KA, Liu JB, Ko CY, Feldman LS, Thacker JK. Adherence to enhanced recovery protocols in NSQIP and association with colectomy outcomes. Ann Surg. 2019;269(3) Retrieved from https://journals.lww.com/annalsofsurgery/Fulltext/2019/ 03000/Adherence_to_Enhanced_Recovery_Protocols_in_NSQIP. 18.aspx.

11. The fourth patient report of the National Emergency Laparotomy Audit 2018 - Full Patient Report.

12. The Royal College of Surgeons of England/Department of Health. The higher risk general surgical patient: towards improved care for a forgotten group. 2011. Available at: http://www.rcseng.ac.uk/ publications/docs/higher-risk-surgicalpatient/.

13. The Royal College of Surgeons. Emergency Surgery: Standards for unscheduled care. 2011. Available at https://www.rcseng.ac.uk/ library-and-publications/rcs-publications/docs/emergency-surgerystandards-for-unscheduled-care/.

14. National confidential enquiry into patient outcome and death. Knowing the risk. A review of the peri-operative care of surgical patients, 2011. Available from www.ncepod.org.uk/2011report2/ downloads/POC fullreport.pdf

15. National confidential enquiry into patient outcome and death (NCEPOD) elective \& emergency surgery in the elderly: an age old problem. 2010. [http://www.ncepod.org.uk/2010eese.htm].

16. The national hip fracture database: part of the falls and fragility fracture audit programme. https://www.nhfd.co.uk . Accessed 25th August 2019.

17. The National Hip Fracture Database Annual Report 2018. Available at https://www.nhfd.co.uk/20/hipfractureR.nsf/docs/2018Report Accessed on 25th August 2019.

18. Perioperative quality improvement programme. https://pqip.org.uk/ content/home.

19. Perioperative quality improvement programme; Annual report 2017-2018. Available at https://pqip.org.uk/pages/ar2018 accessed on 30th August 2019.

20. Perioperative quality improvement programme; Annual report 2018-2019. Available at https://pqip.org.uk/pages/ar2019. Accessed on 1st Oct 2019. PQIP report highlighting enhanced recovery as a key focus for $\mathbf{2 0 1 9 / 2 0 2 0}$.

21. POM VLAD: https://pqip.org.uk/pages/4. Accessed on 30th Aug 2019.

22.• Royal College of Anaesthetists. Perioperative medicine: a pathway to better surgical care. Available at https://www.rcoa.ac.uk/sites/ default/files/PERIOP-2014.pdf accessed on 30th Aug 2019. The RCoA Perioperative Medicine Programme.

23. Video available at https://www.rcoa.ac.uk/periopmed/animation. Accessed on 30th August 2019.

24. Engelman DT, Ben Ali W, Williams JB, et al. Guidelines for perioperative care in cardiac surgery: enhanced recovery after surgery society recommendations. JAMA Surg. Published online May 04, 2019. 154(8):755-66. https://doi.org/10.1001/jamasurg.2019.1153.

25. Bulletin; Magazine for members of the Royal College of Anaesthetists. September 2019:29-43.

26. NHS digital. Patient related Outcome mEasures (PROMs) for hip and knee replacements. https://digital.nhs.uk/data-and-information/ publications/statistical/patient-reported-outcome-measures-proms/ for-hip-and-knee-replacement-procedures-april-2018-to-march2019/content. Accessed on 30th Aug 2019. 
27. Oliver A, Greenberg CC. Measuring outcomes in oncology treatment: the importance of patient-centered outcomes. Surg Clin North Am. 2009;89(1):17-25, vii. https://doi.org/10.1016/j.suc. 2008.09.015.

28. Rabin R, de Charro F. EQ-5D: a measure of health status from the EuroQol group. Ann Med. 2001;33(5):337-43.

29. WHODAS 2: https://www.who.int/classifications/icf/whodasii/en/ index6.html.

30. Manchester ERAS plus programme. https://www.erasplus.co.uk accessed on 10th September.

31. ERAS plus manual available at https://www.erasplus.co.uk/pdf/ Eras-GM-Manual.pdf. Accessed on 25th Aug 2019.

32. Macmillan Cancer Support: prehabilitation for people with cancer; principles and guidance for prehabilitation within the management and support of people with cancer. Available at https://www. macmillan.org.uk/assets/prehabilitation-guidance-for-people-withcancer.pdf. Accessed on 15th Aug 2019. Macmilliam guidelines on prehabilitation.

33. West MA, Loughney L, Lythgoe D, Barben CP, Sripadam R, Kemp GJ, et al. Effect of prehabilitation on objectively measured physical fitness after neoadjuvant treatment in preoperative rectal cancer patients: a blinded interventional pilot study. Br J Anaesth. 2015;114(2):244-51. https://doi.org/10.1093/bja/aeu318 Benefits of prehabilitation in cancer surgery, the pilot study.

34. Moran J, Guinan E, McCormick P, Larkin J, Mockler D, Hussey J, et al. The ability of prehabilitation to influence postoperative outcome after intra-abdominal operation: a systematic review and meta-analysis. Surgery. 2016;160(5):1189-201 Meta-analysis of the effects of prehabilitation on outcomes.

35. Chen Y, Ahmad M. Effectiveness of adjunct psychotherapy for cancer treatment: a review. Future Oncol. 2018;14(15):1487-96.
36. Hoon LS, Sally CWC, Hong-Gu H. Effect of psychosocial interventions on outcomes of patients with colorectal cancer: A review of the literature. 2013;17(6):883-91.

37. Rodriguez-Larrad A, Lascurain-Aguirrebena I, AbeciaInchaurregui LC, Seco J. Perioperative physiotherapy in patients undergoing lung cancer resection. Interact Cardiovasc Thorac Surg. 2014;19(2):269-81.

38. Crandall K, Maguire R, Campbell A, Kearney N. Exercise intervention for patients surgically treated for non-small cell lung Cancer (NSCLC): a systematic review. Surg Oncol. 2014;23(1):17-30.

39. Padilha, Marinello PC, Galvao DA, Newton RU, Borges FH, Frajacomo F, et al. Evaluation of resistance training to improve muscular strength and body composition in cancer patients undergoing neoadjuvant and adjuvant therapy: a meta-analysis. J Cancer Surviv. 2017;11(3):339-49.

40. Arends J, Bachmann P, Baracos V, Barthelemy N, Bertz H, Bozzetti F, et al. (2017) ESPEN guidelines on nutrition in cancer patients. Clin Nutr. 2017;36(1):11-48.

41. Agarwal DK, Viers BR, Rivera ME, et al. Physical activity monitors can be successfully implemented to assess perioperative activity in urologic surgery. Mhealth. 2018;4:43. Published 2018 Sep 26. https://doi.org/10.21037/mhealth.2018.09.05.

42. Daskivich TJ, Houman J, Lopez M, et al. Association of wearable activity monitors with assessment of daily ambulation and length of stay among patients undergoing major surgery. JAMA Netw Open. 2019;2(2):e187673. Published 2019 Feb 1. https://doi.org/10.1001/ jamanetworkopen.2018.7673.

Publisher's Note Springer Nature remains neutral with regard to jurisdictional claims in published maps and institutional affiliations. 